\title{
A Multiwavelength Study of Barnard 1: A Young Star-forming Region
}

\author{
Srabani Datta* \\ Jodrell Bank Centre for Astrophysics, University of Manchester, Oxford Road, Manchester M13 \\ 9PL, U.K. \\ Email- Srabani.Datta@manchester.ac.uk
}

\begin{abstract}
We perform a multiwavelength study of a young star-forming region in Perseus, Barnard 1, involving data in the infrared using the Infrared Array Camera (IRAC) and the Multiple Imaging Photometer (MIPS) on the Spitzer Space Telescope from the Cores 2 Disc (C2D) programme and the 2 Micron All Sky Survey (2MASS); in the sub-mm using the Submillimeter Common User Bolometer Array (SCUBA) on the James Clerk Maxwell telescope (JCMT). We detect huge plume-like outflows from very young stars around the region, probably connected to it. We also determine age, mass and extinction of these young objects and derive dust and molecular properties.
\end{abstract}

From Planets to Dark Energy: The Modern Radio Universe $1^{\text {st }}-5^{\text {th }}$ October 2007

The University of Manchester, United Kingdom

${ }^{*}$ Speaker. 


\section{Introduction}

Barnard 1 is a dark cloud in the Perseus molecular cloud complex. It is located at a distance of $320 \mathrm{pc}$ [1] at RA $3 \mathrm{~h} 33 \mathrm{~m} \mathrm{14.09s;31d} \mathrm{8m} \mathrm{59.0s} \mathrm{[2].} \mathrm{It} \mathrm{is} \mathrm{connected} \mathrm{to} \mathrm{L1448,} \mathrm{L1455,} \mathrm{NGC} \mathrm{1333,}$ B5 and IC348 in the Perseus complex through an ambient molecular gas of density about $10^{2} \mathrm{~cm}^{-2}$. It has a dense core surrounded by a less dense envelope and a thin outer shell.

Observations reveal that B1 contains a clumpy substructure, several young stellar objects (YSOs) and Herbig Haro (HH) objects [1,2,3] around it's central region. Early star formation can be traced by Herbig Haro objects, of which 26 are found in B1 [1]. We have found parsec scale outflows from MIPS images (Fig.1), which implies that star formation has been taking place for at least 10,000 years.

\section{Extinction studies in B1}

Our analysis of early star formation in B1 consists of constructing a K-band luminosity function and measuring the extinction in the K-band $(2.15 \mu \mathrm{m})$. We use images from the Spitzer Infrared Array Camera (IRAC) and Multiple Imaging Photometer (MIPS) instruments. IRAC images are in the mid-infrared in $3.6 \mu \mathrm{m}, 4.5 \mu \mathrm{m}, 5.8 \mu \mathrm{m}$ and $8.0 \mu \mathrm{m}$, while the MIPS images are in 24 $\mu \mathrm{m}$. The processed data (post BCD) were taken from the Cores 2 Disc (C2D) Spitzer archive using Leopard (Table 1).

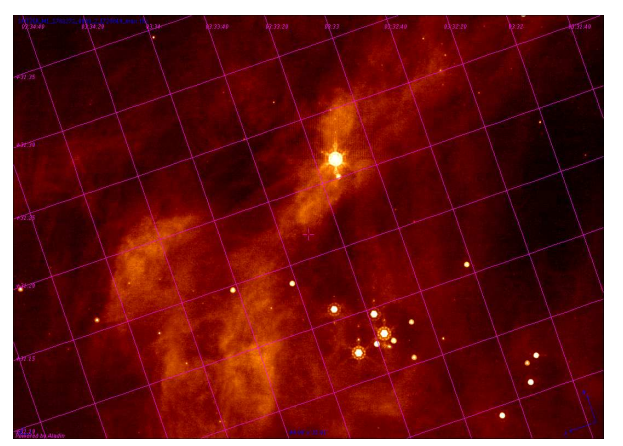

Figure 1: Spitzer MIPS image of Barnard 1 showing parsec scale outflows (AOR 5782272).

Table 1: Details of IRAC and MIPS data taken from the Spitzer archives.

\begin{tabular}{|c|c|c|c|c|}
\hline AOR & RA & Dec & Instrument & PID \\
\hline 5782272 & $3 \mathrm{~h} 33 \mathrm{~m} 34.0 \mathrm{~s}$ & $31 \mathrm{~d} \mathrm{08m} \mathrm{57.0s}$ & MIPS & 178 \\
5785344 & $3 \mathrm{~h} 33 \mathrm{~m} 36.0 \mathrm{~s}$ & $31 \mathrm{~d} \mathrm{08m} \mathrm{50.0s}$ & IRAC & 178 \\
5788928 & $3 \mathrm{~h} 33 \mathrm{~m} 34.0 \mathrm{~s}$ & $31 \mathrm{~d} 08 \mathrm{~m} \mathrm{57.0s}$ & MIPS & 178 \\
5792512 & $3 \mathrm{~h} 33 \mathrm{~m} 17.4 \mathrm{~s}$ & $31 \mathrm{~d} 09 \mathrm{~m} 36.0 \mathrm{~s}$ & IRAC $_{\text {map }}$ & 178 \\
\hline
\end{tabular}

We look for embedded stellar clusters in the dusty regions of B1, which would otherwise be obscured in visible wavelengths. Our methods are statistical, based on stellar surface density 
studies. We derive the $A_{k}$. values using $\mathrm{H}-\mathrm{K}$ colours of all stars taken from 2MASS data as the $\mathrm{K}$ band is most sensitive to embedded young stars. We reject stars with $H-K$ values greater than $3 \sigma$ from the mean in order to avoid foreground and background stars [4]. Fig.2 shows a $A_{k}$ contour map over the MIPS field of view (AOR 5782272).

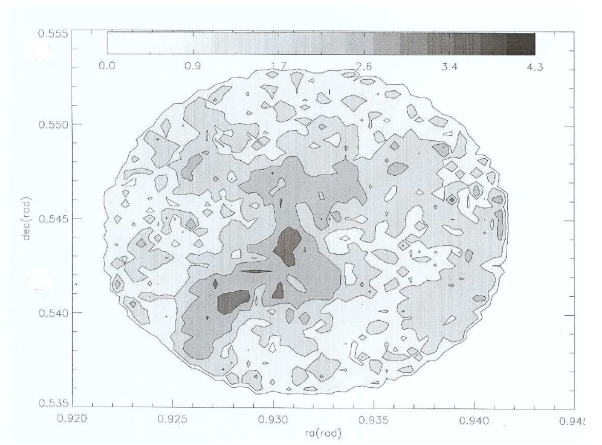

Figure 2: Map of $A_{k}$ in the area given in MIPS AOR 5782272 derived from 2MASS data of all stars from H-K colors.

We also construct the K-band luminosity function (KLF), looking for significant asymmetric structure (figure 3). Our results show that the B1 region is dusty with predominantly young stars and YSOs.

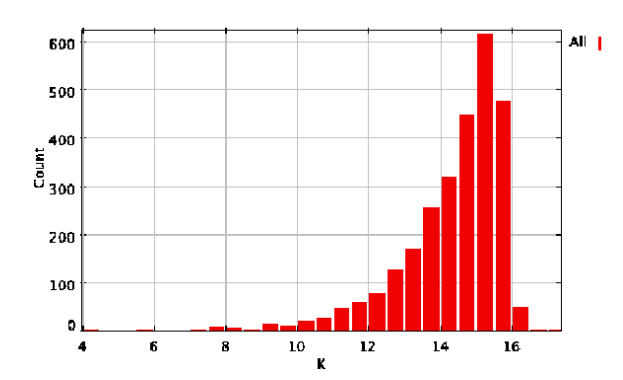

Figure 3: K-band apparent magnitude histogram for B1 for all stars in MIPS image (AOR 5782272).

The author would like to thank the Spitzer Science Centre for the data taken from it's archives.

\section{References}

[1] Walawander,J; et. al. 2005 Ap.J.; 130; 1795

[2] Matthews,B.C. \& Wilson, C.D. 2002; Ap.J.;574; 822

[3] Walawander,J ;Bally, J. \& Reipurth, B. 2005;A.J.; 129;2308

[4] Gutermuth,R.A.; et. al. 2005; Ap.J.; 632;397 